\title{
Las intersexualidades en la educación afectivo-sexual: análisis sobre su presencia y tratamiento
}

\author{
Alejandro Granero Andújar \\ Universidad de Huelva. España. \\ alejandrograneroandujar@gmail.com \\ Teresa García Gómez \\ Universidad de Almería. España. \\ tgarcia@ual.es
}

Recibido: 6/5/2019

Aceptado: $16 / 1 / 2020$

Publicado: 20/7/2020

\section{Resumen}

El presente trabajo pretende conocer en profundidad el grado de presencia de las intersexualidades y el tratamiento recibido en la educación afectivo-sexual, teniendo en cuenta que es una realidad educativa escasamente explorada. Para ello hemos realizado un estudio de caso colectivo en dos institutos de educación secundaria obligatoria de Almería. Las técnicas de recogida de datos empleadas han sido la observación no participante, la entrevista semiestructurada en profundidad y el análisis documental. Los datos obtenidos se han analizado mediante la técnica de codificación. Los resultados constatan que las intersexualidades constituyen una realidad prácticamente desconocida y olvidada en los contextos educativos estudiados, tanto por su omisión como por la superficialidad con la que son abordadas, y que reproducen valores excluyentes y discriminatorios, como el binarismo y la monosexualidad, que han sido interiorizados por el alumnado participante, apareciendo en él actitudes excluyentes y no igualitarias. Concluimos que es necesario abordar de manera trasversal y profunda las intersexualidades en el mundo académico, a fin de no reproducir valores discriminatorios y trabajar para la inclusión de la diversidad de corporalidades existentes. Asimismo, consideramos fundamental potenciar el debate sobre la normatividad imperante y que ello permita cuestionar las concepciones binaristas y monosexistas en las prácticas docentes.

Palabras clave: currículo oculto; diversidad corporal; educación secundaria; educación afectivo-sexual; intersexualidad

Resum. Les intersexualitats en l'educació afectiva i sexual: anàlisi sobre la presència $i$ el tractament

El present treball pretén conèixer en profunditat el grau de presència de les intersexualitats i el tractament rebut en l'educació afectiva i sexual, tenint en compte que aquesta és una realitat educativa escassament explorada. Per això hem realitzat un estudi de cas col-lectiu en dos instituts d'educació secundària obligatòria d'Almeria. Les tècniques de recollida de dades emprades han estat l'observació no participant, l'entrevista semiestructurada en profunditat i l'anàlisi documental. Les dades obtingudes s'han analitzat mitjançant la 
tècnica de la codificació. Els resultats constaten que les intersexualitats constitueixen una realitat pràcticament desconeguda i oblidada en els contextos educatius estudiats, tant per l'omissió com per la superficialitat amb què són tractades, i que s'hi reprodueixen valors excloents i discriminatoris, com ara el binarisme i la monosexualitat, que han estat interioritzats per l'alumnat participant, qui ha manifestat actituds excloents i no igualitàries. Concloem que cal tractar de manera transversal i profunda les intersexualitats en el món acadèmic, per tal de no reproduir valors discriminatoris i treballar per incloure-hi la diversitat de corporalitats existents. Així mateix, considerem fonamental potenciar el debat sobre la normativitat imperant i que això permeti qüestionar les concepcions binàries i monosexistes en les pràctiques docents.

Paraules clau: currículum ocult; diversitat corporal; educació secundària; educació afectiva i sexual; intersexualitat

\section{Abstract. Analysis of the presence and approaches to intersexuality in emotional and sexual education}

Intersexuality is an underexplored educational reality. This work aims to determine the extent to which intersexuality is present in emotional and sexual education and how it is approached. To this end, a multiple case study was carried out in two secondary education schools in Almeria. The data were collected by means of non-participant observation, in depth semi-structured interviews and documentary analysis and analysed using a coding technique. The results reveal that intersexuality is a virtually unknown reality that is largely ignored in this educational context due both to omission and the superficiality with which the topic is addressed. Additionally, this approach to intersexuality reproduces excluding and discriminatory values, such as binarism and monosexuality, which have been internalized by the participating students, thus giving rise to exclusionary and unequal attitudes among them. We conclude that it is necessary to address intersexuality in educational contexts in a transversal and profound manner in order not to reproduce discriminatory values and to promote the inclusion of diverse corporealities. Similarly, it is essential to discuss the prevailing concept of normativity in greater depth and question binarist and monosexist conceptions in teaching practices.

Keywords: hidden curriculum; body diversity; secondary education; affective-sex education; intersexuality

\section{Sumario}

\section{Introducción}

2. Marco teórico

3. Estado de la cuestión

4. Objetivos

5. Metodología

6. Participantes y contextos

de investigación
7. Instrumentos de recogida de datos

8. Procedimiento de análisis y autenticidad de los datos

9. Resultados

10. Conclusiones

Referencias bibliográficas 


\section{Introducción}

La escuela, como institución inmersa en el tejido sociocultural, no es ajena a la situación que atañe a las intersexualidades, pues las discriminaciones y las exclusiones también se encuentran presentes en los contextos educativos.

García (2015) mostraba esta como una herramienta para la conformación e interiorización de la identidad y los valores de género, con lo que contribuía a la percepción dualista de la sexualidad. Asimismo, el binarismo que demarcan sus espacios (como los baños o los vestuarios) impide que las personas intersexuales encuentren su lugar en ella, puedan conformar un sentimiento identitario educativo y consigan resguardarse de la lectura "monstruosa» que realiza la comunidad escolar de sus cuerpos (Fausto-Sterling, 1993).

Jones y Hillier (2012) también visibilizaron la presencia de discursos binaristas en la educación afectivo-sexual impartida en algunos centros educativos australianos, complementados a su vez con una perspectiva excluyente de las identidades y de las corporalidades lesbianas, gais, trans, intersexuales y queers (LGTBIQ) por su omisión entre los contenidos abordados.

Más allá del carácter normalizador de sus espacios y contenidos, la crueldad escolar constituye una realidad que convierte los contextos educativos en espacios hostiles para quienes transgreden las fronteras de la sexualidad y los conforman en dispositivos de reproducción de los valores normativos imperantes. En esta línea, Jones y Hillier (2013) señalaron que el 81\% de los abusos sufridos por la juventud del colectivo LGTBIQ se producían en centro educativos.

Entre las consecuencias que generan estas violencias, rechazos y discriminaciones, encontramos que las escuelas son percibidas como lugares inapropiados entre gran parte del alumnado LGTBIQ, por lo que el 25\% de este evita la asistencia a ellas, ante la incesante presión de cumplir con los estereotipos y las normas dominantes (Jones et al., 2016).

Por último, hallamos que las investigaciones implementadas sobre intersexualidades en el ámbito educativo muestran la existencia de desinterés e indiferencia por parte de los centros educativos (Jones, Gray y Harris, 2014), así como del personal trabajador de estos (Jackson, 2009; Jones et al., 2014; Luke, Goodrich y Scarborough, 2011; Perkins, 2012). Asimismo, los materiales didácticos resultan excluyentes sobre las formas de corporalidad no binarias (España, 2015; Potgieter y Reygan, 2012).

No obstante, los aportes científicos también visibilizan la implicación del profesorado y de la comunidad educativa como eje transformador de las instituciones escolares en lugares de inclusión, respeto e igualdad (Baez y Del Cerro, 2012; Escobar, 2013).

\section{Marco teórico}

Hablamos de intersexualidades, término originario de Goldschmidt (1917), para referirnos a personas cuyas características fisiológicas no se corresponden con los rasgos definitorios de las categorías de sexo binarias: macho o hembra. 
La bióloga Fausto-Sterling (2006), mediante el estudio de los nacimientos con características sexuales no binarias, concluyó que las posibilidades de configuración fisiológica van más allá de las dos opciones binarias (macho o hembra). Dentro de este abanico de posibilidades, las dos categorizaciones binarias quedarían en los extremos, comprendiendo entre sí un rango de diversidad integrada en lo que conocemos como intersexualidades. Así, las personas intersexuales rompen con la linealidad de los requisitos determinados en dicho modelo de categorización basado en criterios biológicos.

Tabla 1. Correlación entre los criterios de asignación de la categoría sexo

\begin{tabular}{llcl}
\hline Categoría & Genitales principales & Cariotipo & Prevalencia hormonal \\
\hline Macho & Testículos y pene & $\mathrm{XY}$ & Andrógenos \\
\hline Hembra & Ovarios y útero & $\mathrm{XX}$ & Estrógenos \\
\hline Intersexual & No existe linealidad entre dichos criterios. & \\
\hline
\end{tabular}

Fuente: elaboración propia.

Sax (2002) afirmaba que en nuestras sociedades la cifra de nacimientos de bebés intersexuales era de 1 entre 4500-5500, prevalencia superior al alumbramiento de personas pelirrojas. Fausto-Sterling (2006) estimó que esta cifra era de un $1,72 \%$. A través de los datos estadounidenses proporcionados por la Intersex Society of North America (ISNA), García (2015) llevó a cabo una extrapolación de las cifras por la cual determinó que el número de personas intersexuales nacidas en España en el año 2012 fue de $227^{1}$.

Por tanto, pese a que la existencia de personas intersexuales conforma una parte poblacional significativa, el modelo normativo hegemónico de clasificación sexual solamente contempla como posibilidades las categorías de macho y hembra, excluyendo e invisibilizando aquellas otras que se encuentran más allá de esta dualidad. Este hecho recibe el nombre de binarismo (García, 2015). Simultáneamente, queda determinado que podemos pertenecer exclusivamente a una de estas categorías, lo que los movimientos activistas intersexuales denominan monosexualidad.

A partir de la lectura binarista y monosexual de los cuerpos, las intersexualidades resultan desestabilizantes del sistema normativo de sexualidades al romper con las bases biologicistas y dualistas que cimientan el resto de los engranajes de este (género, roles de género e identidad afectivo-sexual) y preservan las fronteras de las categorías sexo-genéricas imperantes. Ello provoca que sean percibidas como la "ambigüedad sexual», lo que genera la incomodidad desestabilizante de los mandatos socioculturales dominantes que dictan cómo deben ser nuestros cuerpos, comportamientos, valores, sentimientos y actitudes (Chase, 2005). Tales lecturas de los cuerpos intersexuales como anormales, patológicos, abyectos y ambiguos asientan el campo de cultivo de los valores interfóbicos.

1. Única cifra aportada sobre nacimientos de personas intersexuales en España. 
Si bien el término interfobia todavía no tiene una definición consensuada en el ámbito académico, emplearemos la siguiente definición para ello:

El rechazo, la discriminación, la invisibilización, la denostación y los diferentes tipos de violencia basados en prejuicios y estigmas hacia las personas con variaciones en las características sexuales que transgreden la idea del binarismo acerca de cómo deben ser los cuerpos. (Parra, 2018, p. 43)

En este sentido, las personas intersexuales se conforman como un sector poblacional que sufre las discriminaciones y violencias generadas por el hecho de quedar fuera de los márgenes del sistema hegemónico sociocultural, al igual que ocurre con las no heterosexualidades, las identidades trans, las personas migrantes y racializadas, las comunidades étnicas no imperantes, el colectivo de personas con diversidad funcional, etc., exponiéndose a lo que Meyer (2003) denominó estrés de minorías. Este concepto, más cercano a una perspectiva psicosocial de causalidad de los trastornos psicológicos, visibiliza los problemas mentales de las personas que conforman los sectores sociales minoritarios como producto del estrés provocado por el rechazo social constante en el tiempo.

Pero si la cifra de nacimientos de personas intersexuales es relativamente significativa para conformar un sector poblacional visible, ¿por qué no encontramos las intersexualidades entre nuestra cotidianeidad? La respuesta a ello la hallamos en tres razones fundamentales.

En primer lugar, debido a que ciertos rasgos intersexuales exteriores no resultan visibles en el momento del nacimiento. Por ello, en muchas ocasiones, las características identificativas de la intersexualidad no son descubiertas a lo largo de la vida, mientras que otras son halladas en la pubertad o la adultez gracias a pruebas médicas, ausencia de desarrollo de caracteres sexuales secundarios propios de la categoría sexual asignada (como inexistencia de menstruación en personas de la categoría de sexo hembra), esterilidad o desarrollo de caracteres sexuales secundarios propios de la otra categoría sexual binaria (tales como desarrollo mamario en personas categorizadas como macho).

La segunda razón viene dada por el llamado proceso de reasignación de sexo normativo, resultante de la forma hegemónica de pensar y entender los cuerpos que orienta el accionar científico-médico-jurídico para ocultar y "corregir» las corporalidades no binarias (García, 2015; Hernández, 2009; Nieto, 2003). Así, en el momento del nacimiento de un bebé intersexual, este es sometido a procesos quirúrgicos y hormonales para adecuar sus características corporales a una de las dos categorías binarias. Asimismo, desde las instituciones y el ámbito médico-científico se elimina cualquier registro de intersexualidad fuera del alcance sanitario, ocultando la verdadera naturaleza de esta persona (García, 2015).

Dichos procesos médicos conllevan frecuentemente altos costes que varían desde problemas físicos, en muchos casos irreversibles, en miembros sanos (dolores, esterilidad, pérdida definitiva de sensibilidad, infecciones periódicas, cicatrices, etc.); hasta psicológicos, como el conflicto con la identidad de géne- 
ro correspondiente al sexo asignado, cuya cifra se sitúa entre el 8,5\% y el 20\% de las personas intervenidas (García, 2015), o experiencias médicas humillantes y vejatorias prolongadas en el tiempo que pueden generar depresiones y traumas, y que concluyen en suicidio en algunas ocasiones (Butler, 2006; Chase, 2005; Nieto, 2003). No obstante, existen países donde tales prácticas ya han sido abolidas, como es el caso de Malta, en 2015, Chile, en 2016, y Portugal, en 2018.

Como tercera y última razón, la invisibilización de las intersexualidades viene provocada por la posibilidad única de registro civil como «hombre»o «mujer», es decir, las opciones de inscripción de sexo ofrecidas por el marco jurídico quedan limitadas a una de las dos categorías binarias. Pese a ello, cada vez más países abren las posibilidades de registro a formas no binarias: Australia, Pakistán, Nepal, Malta y, recientemente, Alemania.

\section{Estado de la cuestión}

A partir de una revisión en las principales bases de datos científicas (Scopus, Dialnet, TESEO, ISOC, ERIC, Education Source, REDIB, OAISTER, Redalyc, OVID Medline y Web of Science) sobre el estado de las intersexualidades en el marco científico-educativo práctico de las etapas educativas de infantil, primaria y secundaria, encontramos una falta de atención desde la investigación a este sector poblacional, hallando únicamente trece investigaciones en el ámbito internacional (Baez y Del Cerro, 2012; Escobar, 2013; España, 2015; Harris y Farrington, 2014; Jackson, 2009; Jones et al., 2014; Jones et al., 2016; Jones y Hillier, 2012, 2013; Luke et al., 2011; Perkins, 2012; Potgieter y Reygan, 2012; Vega, Crawford y Van Pelt, 2012).

Esta carencia se acentúa en el contexto español, con tan solo una de estas investigaciones (España, 2015). El resto de ellas pertenecen a países como Argentina, Colombia, Canadá, Estados Unidos, Australia o Sudáfrica. Con ello se hace notable el desconocimiento científico y académico sobre nuestro objeto de estudio en el Estado español.

Entre los resultados presentados en nueve de las trece investigaciones encontradas, esta carencia se ve agravada al integrarse las intersexualidades de manera generalizada en una categoría de naturaleza más colectiva (como "LGTBI», "LGTBIQ», «trans-spectrum», "gender-diverse», etc.). De este modo, las carencias, necesidades o situaciones de las intersexualidades en la práctica educativa se ven desvanecidas entre el conjunto de los resultados sobre identidades afectivo-sexuales y de género, invisibilizándose así la información individual, concreta y específica sobre ellas.

\section{Objetivos}

Mediante este trabajo nos proponemos conocer en profundidad el grado de presencia de las intersexualidades y el tratamiento recibido en la educación afectivo-sexual ante la carencia de estudios existentes sobre estas en la dimen- 
sión educativa. Para ello hemos establecido objetivos específicos que nos han permitido determinar de manera más concreta el fin a alcanzar:

- Conocer el grado de presencia de las intersexualidades en el marco de la educación afectivo-sexual del Programa Forma Joven ${ }^{2}$ desarrollado en dos institutos de educación secundaria (IES).

- Identificar las posibles prácticas discriminatorias y excluyentes de las intersexualidades en las actividades realizadas en la educación afectivo-sexual del Programa Forma Joven implementado en dos IES y las personas participantes.

- Llevar a cabo una interpretación sobre las posibles repercusiones en el alumnado del abordaje de las intersexualidades en las prácticas estudiadas.

- Aportar conocimiento a un campo vagamente conocido en la actualidad, como es el caso de las intersexualidades en el ámbito educativo, que permita repensar y reconfigurar las prácticas docentes a partir de la situación y de las necesidades existentes.

\section{Metodología}

Para el logro de los objetivos propuestos en este trabajo, hemos decidido emplear la metodología interpretativa. Esta es la actividad de carácter sistemático que busca comprender en profundidad los fenómenos sociales y educativos para la conformación de un cuerpo organizado de saberes, sin olvidar la intención transformadora de las prácticas y de los escenarios estudiados (Sandín, 2003). Todo ello mediante la inmersión, la interacción, la comprensión y la interpretación en el campo y en las personas involucradas en él a partir de planteamientos o posibles problemas que puedan existir (Cisterna, 2005; Stake, 1999; Taylor y Bodgan, 1992).

Dentro del paradigma interpretativo, decidimos emplear el estudio de caso colectivo (Stake, 1999) de enfoque etnográfico como estrategia de indagación, a fin de alcanzar conocimientos sobre nuestro objeto de estudio, partiendo de la observación y de la comparación de las similitudes y diferencias entre los casos que permitan una comprensión profunda (Coller, 2000). Con ello hemos podido conocer las especificidades de nuestros contextos singulares y comunes, a la par que favorecer la transferencia de los resultados alcanzados a otros contextos según el grado de similitud con nuestros casos y modificar generalizaciones preestablecidas (Guba, 1989; Stake, 1999).

2. El Programa Forma Joven está desarrollado desde el curso 2001-2002 por la Consejería de Salud, la Consejería de Educación, la Consejería de Igualdad y Políticas Sociales y la Consejería de Gobernación de la Junta de Andalucía. Tiene como objetivo promover actitudes y entornos saludables entre la juventud andaluza. 


\section{Participantes y contextos de investigación}

Nuestro trabajo de investigación se ha centrado en los dos casos estudiados: IES El Camino y El Valle ${ }^{3}$. Ambos institutos se encuentran ubicados en la ciudad de Almería y cuentan con una amplia trayectoria en el desarrollo del programa, siendo prácticamente pioneros en su implementación. Tal característica se constituyó como factor sustancial para su selección como casos de estudio.

El primero de ellos alberga a alumnado de nivel socioeconómico bajomedio, presenta una gran diversidad racial y étnica con alumnado payo, gitano y de origen marroquí. Por otro lado, el IES El Valle, situado en un contexto socioeconómicamente desfavorecido, cuenta con altos niveles de alumnado gitano y de procedencia marroquí, siendo la población paya inexistente.

En ellos han participado las personas encargadas de la coordinación y desarrollo de Forma Joven: personal sanitario que impartía los talleres, profesorado coordinador del Programa en el centro y dirección. También se ha contado con el alumnado de los distintos grupos de segundo, tercero y cuarto de ESO, por ser estos cursos a los que se dirigía el Programa.

\section{Instrumentos de recogida de datos}

Como técnicas de recogida de datos hemos llevado a cabo un total de 23 observaciones no participantes de los talleres impartidos con los grupos de segundo, tercero y cuarto de ESO; 43 entrevistas semiestructuradas en profundidad a las personas participantes (personal sanitario que impartía los talleres, profesorado coordinador del Programa en los IES, dirección y alumnado participante); así como análisis documental de los recursos empleados en las prácticas investigadas, con un total de 13 documentos textuales y gráficos analizados (vídeos, diapositivas y folletos utilizados durante los talleres). La recogida de datos se realizó durante los cursos 2013-2014 y 2014-2015.

\section{Procedimiento de análisis y autenticidad de los datos}

Partiendo del enfoque interpretativo de nuestra investigación, hemos empleado la codificación como técnica de análisis. Para ello hemos procedido a la identificación de núcleos de ideas comunes que han establecido las categorías de nuestra investigación para, posteriormente, conformar agrupaciones que permitiesen realizar el análisis de los sentidos grupales, las discrepancias y las interrelaciones entre los datos que nos proporcionasen conocimientos, comprensiones y un entendimiento holístico de nuestro objeto de estudio (Simons, 2011). Así, se han seguido tres pasos principales durante el proceso de análisis:

- Categorización: selección de los tópicos existentes entre los datos que compartían entre sí patrones comunes y elementos relacionados. De este modo

3. A fin de preservar el anonimato de los IES, dichos nombres son ficticios. 
se generaron categorías y subcategorías, siendo la diferencia entre ambas que las primeras denotaban un tópico en sí mismas, mientras que las segundas hacían referencia a aspectos concretos de dichos tópicos (Cisterna, 2005).

- Elaboración emergente de categorías y agrupación de los datos: unión y organización de los datos del mismo tópico en categorías y subcategorías. Durante este proceso surgieron nuevos tópicos y relaciones que conformaron categorías emergentes.

- Análisis holístico de los datos de cada categoría, así como aquellos que tenían relación entre estas, lo que nos permitió alcanzar comprensiones y conocimientos conjuntos de cada categoría o eje temático.

Tabla 2. Categorías establecidas en nuestro proceso de análisis de datos

\begin{tabular}{|c|c|c|c|}
\hline Categorías & Códigos & Subcategorías & Códigos \\
\hline \multirow[t]{4}{*}{ Binarismo } & \multirow[t]{4}{*}{$\mathrm{B}$} & Ausencia de intersexualidades & $\mathrm{BAI}$ \\
\hline & & Limitación de opciones binarias & $\mathrm{BLOB}$ \\
\hline & & Patologización de intersexualidades & $\mathrm{BPI}$ \\
\hline & & Anormalidad de intersexualidades & $\mathrm{BAl}^{*}$ \\
\hline \multirow[t]{2}{*}{ Monosexualidad } & \multirow[t]{2}{*}{ M } & Unicategorización sexual & MUS \\
\hline & & Intersexualidades como indefinidas & MII \\
\hline \multirow[t]{3}{*}{ Interfobia } & \multirow[t]{3}{*}{ I } & Burlas & IB \\
\hline & & Asco & IA \\
\hline & & Miedo & IM \\
\hline \multirow[t]{2}{*}{ Desinformación } & \multirow[t]{2}{*}{$\mathrm{D}$} & Ausencia de conocimientos & DIAC \\
\hline & & Mitos y conceptos erróneos & DIMCE \\
\hline
\end{tabular}

Fuente: elaboración propia.

Para aportar veracidad a nuestro trabajo, hemos empleado tres técnicas de triangulación:

- Metodológica (Taylor y Bodgan, 1992), mediante el análisis contrastado de los datos obtenidos con las tres técnicas de recogida de información.

- De fuentes de datos (Simons, 2011; Stake, 1999), que nos permitió conocer las coincidencias y las diferencias de la información aportada por las distintas fuentes consultadas.

- De investigadores (Simons, 2011; Stake, 1999), gracias a la comparación de las reflexiones, los resultados y las percepciones surgidas entre las personas autoras de este trabajo. 


\section{Resultados}

Las intersexualidades, dentro de las actividades implementadas en el marco de la educación afectivo-sexual de nuestros casos investigados, constituían una realidad poco o nada presente. Este hecho contribuía al binarismo por su omisión entre los contenidos abordados en la mayoría de talleres, así como por el trabajo superficial y breve de aquellas en otros pocos.

En el IES El Camino el trabajo se limitaba a un momento puntual de los talleres de segundo de ESO. Mientras tanto, en el IES El Valle su presencia era nula entre los contenidos planificados, puesto que se trató una única vez tras la pregunta de un estudiante sobre si «una mujer puede nacer con pene», lo que demuestra a su vez que el alumnado mantenía interés por conocer las corporalidades que van más allá de las categorías binarias.

En ambos casos, el trabajo sobre las intersexualidades se limitó a las explicaciones sobre qué son las intersexualidades desde una perspectiva cromosómica (sin atender al abordaje crítico, social, sexual, afectivo, emocional, etc.), resultando discriminante el hecho de que estas fueron mostradas como indefinidas, anormales y patológicas:

Pueden nacer personas con anomalías. (Observación de 3. B, El Valle)

Cuando nacemos tenemos un sexo, hombre o mujer, pero hay personas que el sexo no está muy definido. Hay personas que tienen cromosomas diferentes y no se sabe muy bien si son hombres o mujeres, y no tienen los órganos bien diferenciados. (Sanitaria. Observación en el taller de 2. $\mathrm{A}$, El Camino)

La necesidad de realizar un abordaje profundo y transversal de las intersexualidades se ponía de manifiesto tras las evidencias de desconocimiento y las concepciones erróneas que mantenía el alumnado. Así, encontramos que una parte mayoritaria no tenía noticia de ellas, puesto que un amplio sector de jóvenes mostró sorpresa al conocer su existencia. Asimismo, una parte manifestó miedo al conocer algunas cifras sobre nacimientos de bebés intersexuales o aspectos acerca de la intersexualidad:

ENTREVISTADOR: Intersexual es que naces con órganos de hombre y de mujer. Yamila: ¡Ay, qué miedo, nena! (Entrevista al alumnado de 4. ${ }^{\circ}$ B, El Valle) ENTREVISTADOR: Puede tener testículos y ovarios.

TERE: ¿Y cómo puede ser eso?

ANA: ¡Qué cosa más rara! (Entrevista al alumnado de 2. ${ }^{\circ} \mathrm{C}$, El Camino)

Complementariamente, una parte destacable del alumnado mantenía mitos y concepciones erróneas sobre las intersexualidades, al confundirlas con transexualidad y bisexualidad, así como al afirmar que:

1. Las intersexualidades ocurren debido a que el bebé cambia de «la placenta de la niña a la del niño». (Entrevista al alumnado de 3. ${ }^{\circ} \mathrm{A}$, El Valle)

2. Se debe a que, durante el coito, «la madre y el padre no se han puesto bien derechos». (Entrevista al alumnado de 4. ${ }^{\circ} \mathrm{B}$, El Valle) 
3. Son síntomas de falta de desarrollo:

Victoria: Salió en la tele hace poco de una persona que no sabían si era chico o chica. No estaba desarrollado.

(Entrevista al alumnado de 4..$^{\circ} \mathrm{A}$, El Camino)

4. Deben tomar medicación para producir leche:

Elia: Pero tiene que tomar unas pastillas para que eche leche por los pezones. (Entrevista al alumnado de $2 .^{\circ} \mathrm{C}$, El Camino)

5. Sus rasgos fenotípicos se encuentran, de manera ambigua, entre los cánones de las dos categorías de sexo binarias:

ENTREVISTADOR: ¿¿Sabéis qué son las personas intersexuales?

Nico: Sí, que tiene una parte de mujer y otra de hombre.

(Entrevista al alumnado de 2. ${ }^{\circ} \mathrm{C}$, El Camino)

AвDEL: Tienes la mitad de niño y la mitad de niña.

(Entrevista al alumnado de $3 .^{\circ} \mathrm{A}$, El Valle)

Más allá del análisis sobre la presencia o ausencia de las intersexualidades, entre los contenidos trabajados encontramos que se transmitió una concepción binarista de las opciones de corporalidad sexual y, por tanto, excluyente de las intersexualidades, al mostrar como únicas posibilidades en la categorización de sexo aquellas correspondientes a las opciones binarias, invisibilizando las intersexualidades entre las características fisiológicas existentes en la realidad humana:

La sanitaria transmite que solamente existen dos sexos, hombres y mujeres. (Observación en el taller de 2. ${ }^{\circ} \mathrm{C}$, El Camino)

Cuando es un feto no se sabe si será niño o niña hasta un determinado momento que se queda el clítoris en las niñas o se alarga y se produce el pene en los niños. (Médica. Observación en el taller de 3. ${ }^{\circ}$ A, El Valle)

Este tipo de binarismo también estuvo presente durante los talleres al visibilizar únicamente las categorías hegemónicas y sus características de manera dualista y dicotómica, relegando a las intersexualidades a la invisibilización:

La sanitaria explica los síntomas de la excitación sexual: en las chicas aumenta el tamaño de los labios mayores y menores y el clítoris. En los chicos se produce una erección. (Observación en el taller de 2. $\mathrm{B}$, El Camino)

Se exponen diferentes dibujos de órganos sexuales (binarios) y las sanitarias piden al alumnado que digan si pertenecen a los hombres o a las mujeres. (Observación en el taller de 3. ${ }^{\circ} \mathrm{C}$, El Valle)

Estos datos se corresponden con la mirada de las personas encargadas de la coordinación y el desarrollo del Programa en nuestros casos estudiados (personal sanitario, dirección y profesorado coordinador), quienes durante sus 
entrevistas no hicieron referencia a las intersexualidades entre los contenidos y los objetivos a trabajar desde Forma Joven. No obstante, sí mencionaron otros, como la alimentación, la anatomía, la masturbación, las relaciones no heterosexuales, los preservativos, los embarazos no deseados, las enfermedades de transmisión sexual, etc.

Igualmente, los resultados previamente expuestos se correspondían con la mirada binarista que mostraban las sanitarias que impartían los talleres. En cuanto al IES El Camino, la propia sanitaria afirmó que el trabajo llevado a cabo sobre intersexualidad se limitaba a definir qué es esta y a exponer las causas cromosómicas que la provocan, dejando de lado las diferentes dimensiones posibles a abordar en un espacio dedicado a la educación afectivo-sexual. Por otro lado, las sanitarias del IES El Valle indicaron que las transexualidades y las intersexualidades no las mencionaban durante los talleres, lo que corroboraba los resultados alcanzados mediante las observaciones y el análisis de los materiales empleados. Durante sus entrevistas también comunicaron que limitaban los aprendizajes anatómicos a los órganos de «hombres y mujeres», relegando a las intersexualidades de los aprendizajes transmitidos al alumnado.

En el mismo sentido, las sanitarias, debido a su influencia profesional, mantenían una percepción del Programa ligada a la anatomía y al trabajo preventivo de ITS y embarazos no deseados, sin prestar atención al resto de temáticas que comprendían Forma Joven.

Desde la mirada receptora de los aprendizajes, los resultados nos muestran que la mayoría del alumnado afirmó no haber trabajado las intersexualidades, añadiendo que este hecho puede provocar que las personas intersexuales no se sientan incluidas en las actividades del Programa:

BEA: Pues nunca se ha hablado de eso.

Bibi: Es verdad. Nunca, nunca.

SAlVa: Nunca.

AmaLia: ¿Y creéis que las personas intersexuales se sienten integradas con lo que dicen?

BEA: Si hay alguien yo creo que no se sentirá muy integrado.

Salva: No.

Amalia: No.

(Entrevista al alumnado de $4 .^{\circ} \mathrm{C}$, El Camino)

Contrariamente, una minoría del alumnado afirmó haberse trabajado las intersexualidades en el marco de los talleres del Programa Forma Joven realizados con $2 .^{\circ}$ de ESO. En el análisis de tal afirmación son relevantes tres aspectos. En primer lugar, que una parte de este alumnado no recordaba qué fue lo abordado sobre intersexualidades, mientras que la otra parte expuso que el trabajo se redujo a un plano superficial mediante la mera explicación de qué es la intersexualidad. En segundo lugar, otra parte transmitió que se partía de una mirada patologizante y biologicista, desde la que las intersexualidades eran expuestas como anomalías. En tercer lugar, cuando se les preguntó por los contenidos trabajados y aprendidos en el Programa, no mencionaron las inter- 
sexualidades, mientras que sí expusieron otros diferentes. En cambio, tras preguntarles de forma explícita sobre ellas como contenido trabajado, respondieron afirmativamente. Desde nuestra interpretación, este hecho supone un indicador del tratamiento superficial, en algunos momentos, e inexistente, en otros, de las intersexualidades, por lo que son percibidas entre el alumnado como contenidos de poco peso e interés dentro de Forma Joven.

En cuanto a las percepciones enmarcadas dentro del binarismo de las personas implicadas en la coordinación y el desarrollo del Programa en nuestros casos, hemos percibido que este se encontraba presente en las sanitarias del IES El Valle, al exponer las opciones binarias como únicas posibilidades de categorización en el nacimiento de un bebé:

Cuando fabricas un feto no sabes si es chico o chica, pero si tú lo sacaras sería una chica siempre. Las células que van a dar lugar son las mismas, nada más que dices «esto va a dar lugar al ovario». (Entrevista sanitarias, El Valle)

La mirada binaria también se encontraba presente entre el alumnado participante de ambos casos, ya que:

1. Percibían a las personas intersexuales como raras o indefinidas, por no pertenecer a las categorías normativas:

ENTREVISTADOR: Son personas que tienen los órganos femeninos y masculinos.

Clara: Qué persona más rara.

(Entrevista al alumnado de $2 .^{\circ} \mathrm{B}$, El Camino)

2. Expusieron la cirugía de reasignación genital como solución a la «ambigüedad» anatómica intersexual, percibiendo como necesaria dicha normalización a los patrones hegemónicos:

ETHAN: Eso se opera y ya está.

(Entrevista al alumnado de 3. ${ }^{\circ}$ Diversificación, El Camino)

RAIMUNDO: Luego te analizan y dicen «este tiene $70 \%$ de hombre». Le quitas los ovarios y ya está, y un hombre otra vez.

(Entrevista al alumnado de $3 .{ }^{\circ} \mathrm{C}$, El Valle)

Los resultados también determinan que se reprodujo monosexualidad en ambos casos estudiados al transmitir que únicamente podemos pertenecer a una de las dos categorías de sexo binarias, siendo así percibidas las intersexualidades como indefinidas:

La sanitaria explica que nacemos con un sexo biológico y que algunas personas no tienen el sexo muy definido, estas son las personas intersexuales. (Observación en el taller de $2 .{ }^{\circ} \mathrm{C}$, El Camino)

Un alumno pregunta si puede nacer una mujer con pene y la médica le comenta que sí, que pueden nacer personas con anomalías y añade que, según los cromosomas, es de un sexo u otro. (Observación en el taller de 3..$^{\circ}$ B, El Valle) 
Consideramos necesario, desde las acciones planteadas, romper con esta concepción limitada y excluyente de la diversidad de opciones de categorización en lo referido al sexo, a fin de no legitimar los pensamientos socioculturales monosexuales que están presentes en el alumnado, tal y como hemos podido comprobar en nuestra investigación. Concretamente, percibimos cómo una parte del alumnado mantenía valores monosexuales al excluir de su mirada la posibilidad de pertenencia a ambas categorías o poseer características biológicas comunes:

Rosa: Es una persona que nace y no se sabe de qué sexo es.

(Entrevista al alumnado de $4 .^{\circ} \mathrm{A}$, El Camino)

Pese a que no se trabajó nada sobre interfobia desde el Programa, debido a la ausencia de las intersexualidades entre los contenidos tratados o la poca profundización en su abordaje, el alumnado sí mostró actitudes de rechazo, asco y burla sobre las intersexualidades, al mismo tiempo que las tacharon de mutaciones, feas y anormales.

\section{Conclusiones}

A partir de los resultados obtenidos, concluimos que las intersexualidades constituían una realidad prácticamente olvidada e invisibilizada en los contextos estudiados, puesto que se exponían las dos categorías binarias de sexo como únicas posibilidades reales, válidas y sanas. En las escasas ocasiones en las que se abordaron las corporalidades no binarias, se partía de una mirada patologizante y discriminatoria que las mostraba como anómalas e indefinidas, al mismo tiempo que limitaban su abordaje a su explicación desde un punto de vista biológico (cromosómico y genital). Por ende, podemos decir que, tal como señaló Foucault (1980), la pedagogía de la sexualidad se ha visto medicalizada en función del saber científico-médico normalizador en nuestros casos estudiados.

Consideramos que esta falta de un abordaje profundo y no estigmatizante de las intersexualidades podría ser consecuencia, desde una comprensión holística de lo macro, del desconocimiento científico-académico señalado en el estado de la cuestión. Por ello es necesario potenciar el debate sobre la normatividad imperante dentro del ámbito educativo que permita cuestionar las concepciones y los dogmas binaristas y monosexistas. Al mismo tiempo, consideramos que los valores binaristas presentes entre las personas implicadas en la coordinación e implementación del Programa pueden ejercer influencia en este al sustentar los valores transmitidos, limitar la capacidad de enfoque en el trabajo realizado y potenciar posibles discriminaciones. Por tanto, resulta también necesario el abordaje académico de las intersexualidades para potenciar el cambio de concepciones, creencias y prácticas que permitan realizar una mirada de la educación afectivo-sexual más inclusiva de la diversidad existente. En caso contrario, el alumnado adquiere una concepción de las posibilidades de sexo biológico limitada a las categorías binarias, que se conforman como 
los únicos tipos de corporalidad existentes y sanas. A su vez, quedan fuera de la «normalidad» o «naturalidad» aquellas diferentes a las mostradas. Este hecho contribuye a la estigmatización de las personas intersexuales y favorece la conformación de ideas y prejuicios binaristas y monosexuales que establecen los cimientos para los valores interfóbicos. Como hemos podido comprobar, tales prejuicios se encuentran presentes en el alumnado participante y son reforzados y legitimados por los discursos transmitidos desde la práctica educativa.

Del mismo modo, las exclusiones y discriminaciones reproducidas en los contextos educativos refuerzan los ideales de normalidad en lo referido al ámbito de la sexualidad desde el modelo imperante, afectando también a las no heterosexualidades, a las identidades trans y a las personas que no se ajustan a los cánones tradicionales de los roles de género. Hecho que se constituye también como la base de prejuicios entre el alumnado que no pertenece al colectivo LGTBI, así como de autopercepciones y consecuencias psicológicas negativas entre el alumnado que sí pertenece a este, tales como problemas de autoestima, rechazo al sistema educativo, aislamiento social, conductas lesivas y de riesgo, depresión e, incluso, suicidio (Almeida et al., 2009; Espelage, Aragon y Birkett, 2008; Platero, 2014).

No es un hecho a olvidar que, tal y como denuncia el Observatorio Español contra la LGTBfobia, las tasas de suicidio entre menores no heterosexuales, trans e intersexuales han aumentado un $60 \%$ en casi la última mitad de siglo. Tal porcentaje se corresponde a una media de 50 jóvenes al año, junto con 950 que lo intentan (EFE, 2016).

A raíz de nuestros resultados, también hallamos que en muchas ocasiones las manifestaciones de interfobia entre el alumnado venían acompañadas por el desconocimiento de las intersexualidades, producto de una falta de atención de estas en los contextos educativos. De este modo, tales manifestaciones se vieron reforzadas por el miedo a lo desconocido, así como por las concepciones socioculturales hegemónicas de binarismo y monosexualidad que sustentan su rechazo y discriminación. Valores contra los que no se trabajó en la realidad estudiada, sino que se vieron reforzados.

Por todo ello resulta necesario atender a las formas de corporalidad no binarias en el marco científico y académico educativo, a fin de potenciar el conocimiento y el debate que permita plantear el análisis y la reflexión de los valores y las concepciones sociales presentes en el alumnado y el marco social. Ello permitirá a las instituciones escolares no continuar actuando como dispositivos de deshumanización y discriminación de las intersexualidades mediante su invisibilización y la legitimación de las violencias presentes en las dimensiones sociales y escolares, para pasar a conformarse como espacios de aprendizaje y consolidación de los derechos humanos y valores igualitarios. Igualmente, un mayor conocimiento contribuiría a atender las demandas de organismos internacionales, como la planteada por la Organisation Intersex International Australia (2012), acerca de integrar las intersexualidades de manera transversal y profunda en las acciones educativas, a fin de reducir los abusos y las violencias sufridas. En definitiva, como afirman Aguirre y Domé- 
nech (2018), la transformación educativa "pasa por conseguir que esas "voces silenciadas" dejen de serlo, que deje de existir ese silencio en ciertos colectivos o grupos» (p. 125).

\section{Referencias bibliográficas}

Aguirre García-Carpintero, A. y Doménech Vidal, A. (2018). Historias que transforman: Narrativas en el aula. Revista Educar, 54(1), 123-143. $<$ https://doi.org/10.5565/rev/educar.916>

Almeida, J.; Johnson, R.M.; Corliss, H.L.; Molnar, B.E. y Azrael, D. (2009). Emotional distress among LGBT youth: The influence of perceived discrimination based on sexual orientation. Journal of Youth and Adolescence, 38(7), 1001-1014. <https://doi.org/10.1007/s10964-009-9397-9>

BAez, J. y Cerro, C.G. del (2012). Las voces juveniles en la construcción curricular: Los géneros en las disciplinas escolares. Ponencia presentada en el VI Congresso de Estudos Sobre a Diversidade Sexual e de Gênero da ABEH. Salvador de Bahía (Brasil), del 1 al 3 de agosto.

Butler, J. (2006). Deshacer el género. Barcelona: Paidós.

Chase, C. (2005). Hermafroditas con actitud: Cartografiando la emergencia del activismo político intersexual. En C. Romero Bachiller, S. García Dauder y C. Bargueiras Martínez (eds.). El eje del mal es heterosexual (pp. 87-108). Madrid: Traficantes de Sueños.

Cisterna Cabrera, F. (2005). Categorización y triangulación como procesos de validación del conocimiento en investigación cualitativa. Theoria, 14(1), 61-71. Recuperado de $<$ http://www.redalyc.org/articulo.oa?id=29900107>.

Coller, X. (2000). Estudio de casos. Madrid: Servicio de Publicaciones del Centro Superior de Investigaciones Sociológicas (CSIC).

EFE (10 de septiembre de 2016). Las alarmantes tasas de suicidio LGBTI en España: Casi 50 jóvenes se quitan la vida cada año y 950 lo intentan. La Sexta. Recuperado de <https://www.lasexta.com/noticias/sociedad/alarmantes-tasas-suicidiolgbti-espana-casi-jovenes-quitan-vida-cada-ano-950-intentan_2016091057d420 120cf2d961f2d443fd.html>.

Escobar, A.L. (2013). El respeto por la diversidad sexual y de género en la Institución Educativa la Gabriela en Bello Antioquia. Trabajo de fin de grado. Recuperado de $<$ http://repositorio.ucm.edu.co:8080/jspui/bitstream/handle/10839/522/ Arturo\%20Leon\%20Escobar\%20Cordoba.pdf?sequence=1>.

España, C. (2015). Diversidad sexual en la educación primaria: Actitudes y análisis de recursos. Trabajo de fin de grado. Recuperado de <http://hdl.handle.net/10902/ 6868>.

Espelage, D.L.; Aragon, S.R. y Birkertt, M. (2008). Homophobic teasing, psychological outcomes, and sexual orientation among high school students: What influence do parents and schools have? School Psychology Review, 37(2), 202-216. Recuperado de <https://www.tandfonline.com/doi/abs/10.1080/02796015.200 8.12087894>.

Fausto-Sterling, A. (1993). The five sexes: Why male and female are not enough. The Sciences, 33(2), 20-24.

<https://doi.org/10.1002/j.2326-1951.1993.tb03081.x>

- (2006). Cuerpos sexuados. Barcelona: Melusina.

Foucault, M. (1980). Microfisica del poder. Madrid: La Piqueta. 
García López, D.J. (2015). Sobre el derecho de los hermafroditas. Barcelona: Melusina. Goldschmidt, R. (1917). Intersexuality and the endocrine aspect of sex. Endocrino$\log y, 1(4), 433-456$. $<$ https://doi.org/10.1210/endo-1-4-433>

Guba, E. (1989). Criterios de credibilidad en la investigación naturalista. En J. GimeNO y A.I. PÉrez (coords.). La enseñanza: Su teoría y su práctica (pp. 148-165). Madrid: Akal.

Harris, A. y Farrington, D. (2014). 'It gets narrower': Creative strategies for rebroadening queer peer education. Sex Education, 14(2), 144-158. <https://doi.org/10.1080/14681811.2013.854203>

Hernández Guanche, V. (2009). Intersexualidad y prácticas científicas: ¿Ciencia o ficción? RIPS, 8(1), 89-102. Recuperado de <http://www.redalyc.org/articulo. oa?id $=38011446008>$.

Jackson, J. (2009). 'Dangerous presumptions': How single-sex schooling reifies false notions of sex, gender, and sexuality. Gender and Education, 22(2), 227-238. <https://doi.org/10.1080/09540250903359452>

Jones, T.; Gray, E. y Harris, A. (2014). GLBTIQ teachers in Australian education policy: Protections, suspicions, and restrictions. Sex Education, 14(3), 338-353. <https://doi.org/10.1080/14681811.2014.901908>

Jones, T.M. y Hillier, L. (2012). Sexuality education school policy for Australian GLBTIQ students. Sex Education, 12(4), 437-454. <https://doi.org/10.1080/14681811.2012.677211>

- (2013). Comparing Trans-Spectrum and Same-sex-Attracted Youth in Australia: Increased Risks, Increased Activisms. Journal of LGBT Youth, 10(4), 287-307. <https://doi.org/10.1080/19361653.2013.825197>

Jones, T.; Smith, E.; Ward, R.; Dixon, J.; Hillier, L. y Mitchell, A. (2016). School experiences of transgender and gender diverse students in Australia. Sex Education, 16(2), 156-171. <https://doi.org/10.1080/14681811.2015.1080678>

Luke, M.; Goodrich, K.M. y Scarborough, J.L. (2011). Integration of the K-12 LGBTQI student population in school counselor education curricula: The current state of affairs. Journal of LGBT Issues in Counseling, 5(2), 80-101. $<$ https://doi.org/10.1080/15538605.2011.574530>

Meyer, I. (2003). Prejudice, social stress, and mental health in lesbian, gay, and bisexual populations: Conceptual issues and research evidence. Psychological Bulletin, 129, 674-697. <https://doi.org/10.1037/0033-2909.129.5.674>

Nieto, J.A. (2003). La intersexualidad y los límites del modelo «dos sexos/dos géneros». En O. Guasch y O. Viñuales (eds.). Sexualidades: Diversidad y control social (pp. 69-104). Barcelona: Edicions Bellaterra.

Organisation Intersex International Australia (2012). Intersex for allies. Recuperado de <https://oii.org.au/allies/>.

Parra Abaúnza, N. (2018). Colección de guías sobre derechos sexuales y reproductivos de las mujeres jóvenes: Sexualidad. Cuerpos, identidades y orientaciones. Islas Canarias: Instituto Canario de Igualdad del Gobierno de Canarias. Recuperado de <http://www3.gobiernodecanarias.org/medusa/ecoblog/bmarsanf/files/2018/11/ guia-4-sobre-derechos-sexuales-y-reproductivos-en-mujeres-jovenes.pdf $>$.

Perkins, C. (2012). How Schools Principals Understand and Respond to Homophobia: A Study of One B.C. Public School District Using Ethnodrama. Tesis doctoral. Van- 
couver (Canadá). University of British Columbia. Recuperado de $<$ https://open. library.ubc.ca/media/download/pdf/24/1.0073260/5>.

Platero Méndez, R.L. (2014). Trans*exualidades: Acompañamiento, factores de salud y recursos educativos. Barcelona: Edicions Bellaterra.

Potgieter, C. y Reygan, F.C. (2012). Lesbian, gay and bisexual citizenship: A case study as represented in a sample of South African Life Orientation textbooks. Perspectives in Education, 30(4), 39-51. Recuperado de <https://www.researchgate. net/publication/317719489_Lesbian_gay_and_bisexual_citizenship_A_case_ study_as_represented_in_a_sample_of_South_African_Life_Orientation_text books $>$.

Sandín Esteban, M.P. (2003). Investigación cualitativa en educación: Fundamentos y tradiciones. Madrid: McGraw-Hill.

SAX, L. (2002). How common is Intersex?: A response to Anne Fausto-Sterling. Journal of Sex Research, 39(3), 174-178. <https://doi.org/10.1080/00224490209552139>

Simons, H. (2011). Estudio de caso: Teoría y práctica. Madrid: Morata.

STAKe, R.E. (1999). Investigación con estudio de casos. Madrid: Morata

TAYLOR, S. y Bodgan, R. (1992). Introducción a los métodos cualitativos de investigación. Barcelona: Paidós.

Vega, S.; Crawford, H.G. y Van Pelt, J.L. (2012). Safe schools for LGBTQI students: How do teachers view their role in promoting safe schools? Equity \& Excellence in Education, 45(2), 250-260.

<https://doi.org/10.1080/10665684.2012.671095> 